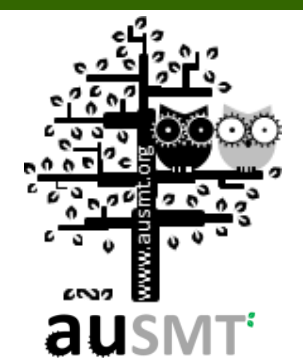

\title{
Ball Nut Preload Diagnosis of the Hollow Ball Screw through Sensed Current Signals
}

\section{Yi-Cheng Huang*, Shi-Lun Sun, and Kuan-Heng Peng}

Department of Mechatronics Engineering, National Changhua University of Education, Taiwan

(Received 17 December 2013; Accepted 28 January 2014; Published 1 September 2014)

*Email: ychuang@cc.ncue.edu.tw

DOI: $10.5875 / a u s m t . v 4 i 3.416$

\begin{abstract}
This paper studies the diagnostic results of hollow ball screws with different ball nut preload levels through the Hilbert-Huang transform (HHT) and multiscale entropy (MSE) process. The method is tested using ball screw pretension and an oil cooling circulation system. MSE was used to determine the hollow ball screw preload status through the servo motor current signals. Ball screws with maximum dynamic preloads of $2 \%, 4 \%$, and $6 \%$ were predesigned, manufactured, and tested. Signal patterns are discussed and revealed by the Hilbert Spectrum. Different preload features are extracted using HHT and MSE. The irregularity development of the ball screw driving motion current can be discriminated and abstracted via MSE based on complexity perception. Experimental results show that the proposed approach can successfully predict the prognostic status of ball nut preload. A comparative evaluation of MSE allows for smart sensing for the health of the ball screw. This method effectively diagnoses the ball nut preload status.
\end{abstract}

Keywords: Ball Nut Preload; Hollow Ball Screw, Oil Cooling System; Hilbert-Huang Transform; Multi-scale Entropy

\section{Introduction}

Precision CNC machines are widely used in modern industry for mass production. Recently, many strategies have been proposed to diagnose machine status, providing operators with important information to extending the machine's useful lifespan. [1] established a system to acquire and analyze vibration signals corresponding to various machine tool states. Calculated results are saved in a database, and a training model is established using Bayesian Classification. Ball screws are widely used in linear actuators for various types of machinery and equipment. Preloading is an effective means to eliminate the backlash and increase the stiffness of the ball screw for precision motion, thus maximizing efficiency [2]. Preload loss leads to a lower natural frequency, lower stiffness, oscillatory positioning, and increased manufacturing downtime. Some methods proposed for tuning preload values are time consuming and incur increased downtime, thus raising the need to predict the ball screw nut preload status during machine operation. Fault diagnosis with most acquired signals requires the assistance of conventional Fourier Transform or Discrete Wavelet Transform in the frequency and time domains. [3] developed an entirely new approach to signal analysis which avoids generating nonphysical results from Complex Trace formalism [4], which defined the concepts of instantaneous amplitude, phase, and frequency such that the original signal can be expressed in terms of a Fourier-like expansion based on these concepts. This process, as well as the definition of instantaneous frequency, worked effectively for mono-component signals. However, for many real applications, the signals are multi-components and are often corrupted by noise. Recently, HHT has been applied to diagnose faults or damage in electrical machines, rolling bearings or tools in mechanical engineering $[5,6]$. 
As mentioned, loss of ball screw preload not only decreases the bandwidth of the frequency response spectrum but also reduces positioning accuracy. Accordingly, industrial mass production applications would benefit from the lifetime prediction for ball screw preload loss status, but few studies have focused on this topic. The Hilbert-Huang transform provides both time and frequency analysis for several engineering applications. However, the complexity of a ball screw with preload in operation is highly nonlinear and non-stationary. Traditional entropy measurements quantify only the regularity (predictability) of a time series on a single scale. No straightforward correlation exists, however, between regularity and complexity. This study applies multiscale entropy analysis [7] to measure the complexity of finite length time series. This computational tool has been applied successfully both to physical and physiological data sets, and can be used with a variety of measures of entropy. Such MSE curves are used to compare the relative complexity of normalized time series. The feature patterns of preload status are determined and abstracted via the complexity of MSE. MSE analysis is used to discriminate the prognostic preload status of ball screws.

\section{Theoretical Background}

\section{The Hilbert-Huang Transform}

In [3], a signal analysis technique based on the decomposition of a signal in terms of empirical modes was introduced by representation within the context of the Complex Trace method introduced by [4]. This method formulates a signal, $X(q)$ ), (q representing either time or a spatial coordinate) as the real part of a complex trace, $Z(q)$,

$$
\mathrm{Z}(q)=\mathrm{X}(q)+\mathrm{iY}(q)
$$

here the imaginary part, $\mathrm{Y}(\mathrm{q})$, is the Hilbert Transform:

$$
\mathrm{Y}(q)=\frac{1}{\pi} P V \int_{-\infty}^{\infty} \frac{X(q)^{\prime}}{q-q^{\prime}} d q^{\prime}
$$

Yi-Chang Huang received his Ph.D in mechanical engineering from Columbia University in 1996. He currently serves as Professor in the Dept. of Mechatronics Engineering at the National Changhua University of Education in Taiwan. His current research interests include Iterative Learning Control Design, health diagnosis of ball screw drive systems and dental hand pieces, and the design of precision piezo-driven stages and controls.

Shi-Lun Sun received his M.S. in mechatronic engineering from the National Changhua University of Education, Taiwan, in 2013.

Kuan-Heng Peng received his M.S. in mechatronics engineering from the National Changhua University of Education, Taiwan, in 2013. where PV indicates the principal value of the singular integral. The complex conjugate pair $(X(q) ; Y(q))$ defines the amplitude, $a(q)$, and phase $\theta(q)$, as an analytical function of the q-variable:

$$
\mathrm{Z}(\mathrm{q})=\mathrm{a}(\mathrm{q}) e^{i(q)}
$$

where

$$
\alpha(q)=\sqrt{x^{2}(q)+Y^{2}}(q)
$$

with the instantaneous frequency defined as:

$$
\omega(q)=\frac{d \theta(q)}{d q}
$$

As previously mentioned, Complex Trace formalism defines the concepts of instantaneous amplitude, phase, and frequency such that the original signal can be expressed in terms of a Fourier-like expansion based on these concepts. This process and the definition of instantaneous frequency work effectively for mono-component signals. However, for many real applications the signals are multi-component and often corrupted by noise. In these situations, the Complex Trace concept fails because the Hilbert Transform processing of those noisy waves generates spurious amplitudes at negative frequencies. Huang et al. developed an entirely new approach to signal analysis to avoid generating nonphysical results. That is, the Hilbert Transform is not directly applied to the signal itself but to each of the members of an empirical decomposition of the signal into intrinsic mode functions (IMFs). These IMFs are individual, nearly mono-component signals with 'Hilbert-friendly' waveforms, to which the instantaneous frequency defined by Equation (4) can be applied.

The algorithm to create the IMFs, referred to as "sifting", is both elegant and simple. First, the local extrema of the data are identified and used to create upper and lower envelopes which enclose the signal completely. These envelopes are used to create a running mean. By subtracting this "mean" from the data, one obtains a new function, which must have the same number of zero crossings and extrema (that is, it exhibits symmetry across the $q$-axis). If the function so constructed does not satisfy this criterion, the "sifting" process continues until an acceptable tolerance is reached [3]. The resulting q-series is the first "IMF", c1(q), and contains the highest frequency oscillations found in the data (i.e., the shortest time scales). The IMF1, is then subtracted from the original data, and this difference R1 is taken as if it were the original signal and then the sifting process is applied to the new signal (R1). 
The process of finding modes, $\mathrm{cj}(\mathrm{q})$, continues until the last mode, the residue $\mathrm{Rn}$, is found which will contain the trend (i.e., the "time-varying" mean). Thus, the signal, $X(q)$, is given by the sum:

$$
x(q)=\sum_{j=1}^{n} \phi(j) \notin n .
$$

The issues of completeness and orthogonality of the IMF-expansion are discussed in [3]. Once the IMFs are obtained, the Hilbert Transform can be applied to each IMF, computing the instantaneous frequency and amplitude using Equations (3) and (4). After applying the Hilbert Transform to each IMF, the signal can be expressed according to Equation (6), where aj(q) and $w j(q)$ are, respectively, the instantaneous amplitude and frequency corresponding to each IMF $c j(q)$.

$$
\mathrm{X}(q)=\operatorname{Re}\left(\sum_{j=1}^{n} a_{j e} i \int w j(q d q)\right)
$$

This expression enables the representation of the instantaneous amplitude and frequency as functions of $q$ in a three-dimensional plot or contour map. The time-frequency representation of the amplitude is named the Hilbert-Huang spectrum, $H(w, q)$.

\section{Multi-Scale Entropy Method}

As stated, MSE analysis is a method for measuring the complexity of finite length time series. This computational tool is applied here with a variety of measures of entropy. In this paper, entropy uses the sample entropy (SampEn) measure [8]. SampEn is a refinement of the approximate entropy [9] family of statistics.

The MSE method incorporates two procedures:

A "coarse-graining" process is applied to the time series. For a given time series, multiple coarse-grained time series are constructed by averaging the data points within non-overlapping windows of increasing length, $\tau$ as shown in Figure 1

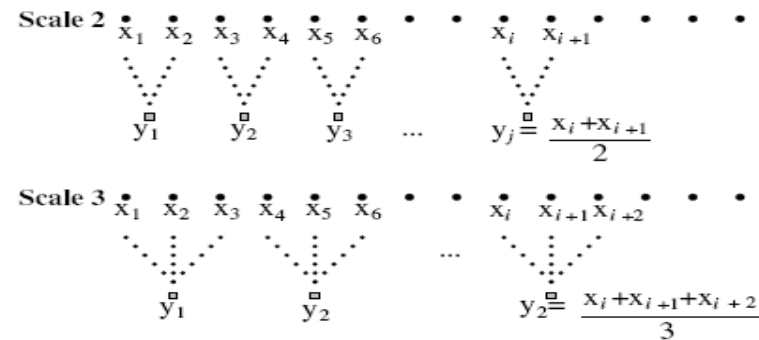

Figure 1. Schematic illustration of coarse-graining procedure for Scales 2 and 3.

Each element of the coarse-grained time series, $y_{j}^{(\tau)}$, is calculated according to Equation (7)

$$
y_{\pi}^{(\tau)}=\sum_{i=(j-1) \tau+1}^{j \tau} x i
$$

where $\tau$ represents the scale factor and $1 \leqq j \leqq N / \tau$. The length of each coarse-grained time series is $N / \tau$. For Scale 1, the coarse-grained time series is simply the original time series.

SampEn is calculated for each coarse-grained time series, and then plotted as a function of the scale factor. SampEn is a "regularity statistic" that "looks for patterns" in a time series and quantifies their degree of predictability or regularity.

In Figure 2, a time series $u[1], \ldots, u[n]$ is shown to illustrate the procedure for calculating sample entropy. Dotted horizontal lines around data points $u[1], u[2]$, and $u$ [3] represent $u[1] \pm r, u[2] \pm r$, and $u[3] \pm r$, respectively. All green points represent data points that match the data point $u[1]$. Similarly, all red and blue points match the data points $u[2]$ and $u[3]$, respectively. The segment shown in Figure 2 involves two green-red sequences, $(u[13], u[14])$ and $(u[43], u[44])$, that match the template sequence $(u[1], u[2])$, but only one green-red-blue sequence that matches the template sequence $(u[1]$, $u[2], u[3])$. Therefore, in this case, the number of sequences matching the 2-component template sequences is two and the number of sequences matching the 3-component template sequence is 1 . These calculations are repeated for the next 2-component and 3-component template sequence, which are, $(u[2], u[3])$

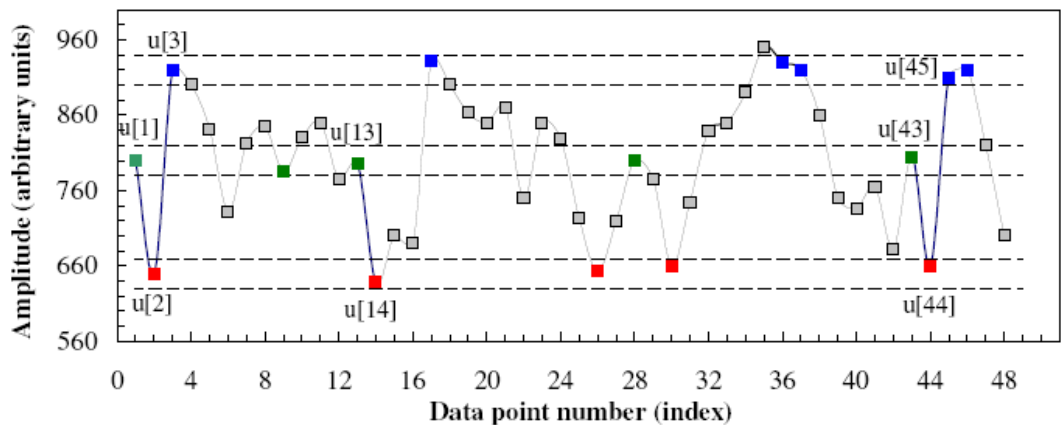

Figure 2. Illustration of time data by applying SampEn. 
and $(u[2], u[3], u[4])$, respectively. The numbers of sequences that match each of the 2 - and 3-component template sequences are again counted and added to the previous values. This procedure is then repeated for all other possible template sequences, $(u[3], u[4], u[5]), \ldots$, $(\mathrm{u}[\mathrm{N}-2], \mathrm{u}[\mathrm{N}-1], \mathrm{u}[\mathrm{N}])$, to determine the ratio between the total number of 2-component template matches and the total number of 3-component template matches. SampEn is the natural logarithm of this ratio and reflects the probability that sequences that match each other for the first two data points will also match for the next point. Therefore, the higher entropy indicates the irregularity and complexity for interested time series data. Here, the viewpoints of preload loss should produce a variety of measures indicating the complexity with high entropy value. The embedded irregularity and complexity of the ball screw describe the nonlinear dynamics when its preload is lost. Intuitively, complexity is associated with meaningful structure richness incorporating correlations over multiple spatiotemporal scales. Preload loss development should exhibit phenomena of more random contact force between balls with the raceway and more irregular force between the balls and ball nut during motion.

\section{Experiment Set Up and Results}

\section{Experiment Set up}

An experimental platform was designed and assembled as shown in Figure 3. This apparatus was fabricated according to the industrial standard for a tapping machine with key specifications as follows: 2kW-3000rpm servo motor (Delta Electronics), oil cooling system of $3 \psi-220 \mathrm{~V}$ with $\pm 2{ }^{\circ} \mathrm{C}$ accuracy. The mechanical structure accuracy is $5 \mu \mathrm{m}$ with a repeated accuracy of 2 $\mu \mathrm{m}$. The maximum positioning speed is $48 \mathrm{M} / \mathrm{min}$ with an acceleration of $1 \mathrm{~g}$ when the motor speed is 3000 rpm.

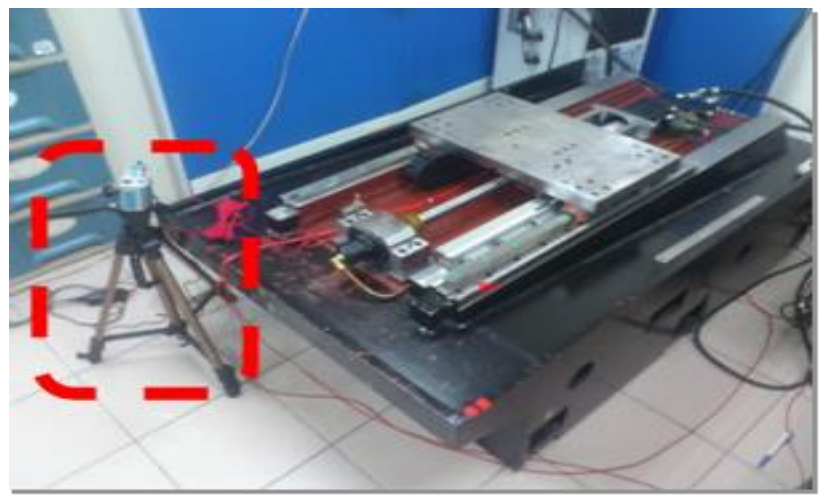

Figure 3. Single axis platform with thermo camera for recording the temperature distribution.

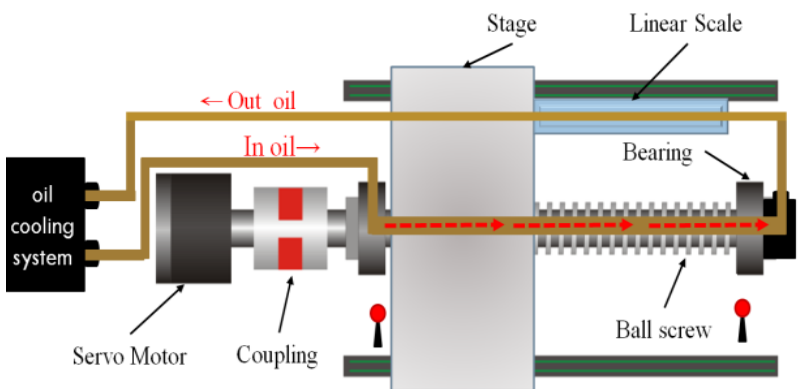

Figure 4. Illustration for the oil cooling circulating system around the hollow ball screw.

The table is set for a traveling distance of $270 \mathrm{~mm}$ with reciprocating movement by varying the motor speed from $300 \mathrm{rpm}$ to $900 \mathrm{rpm}$, and is driven by a servomotor followed by a coupler and a single nut hollow ball screw of Hiwin R-36-16(K3)-FSC-C1-527855-0.006-H with different ball nut preload and pretension levels. The standard ball screw preload force is determined to be $4 \%$ of its maximum dynamic force of 3750 kgf. Its maximum static force is $9542 \mathrm{kgf}$. In this study, the preload percentage of the maximum dynamic force was divided into $2 \%, 4 \%$ and $6 \%$. The constant speed, acceleration, and $\mathrm{S}$ curve were controlled by a Computerized Numerical Control (CNC) controller (model LNC-M310i-V) with feedback from a Heidenhain (C) Encoder Strip. A data acquisition device with a sample rate was used to extract the servo motor current through a PC-based system.

In general, for a CNC feeding table, the specification of the ball screw preload is chosen when the positioning accuracy is determined a priori. In this study, $4 \%$ preload was treated as the standard preload for typical precision motion for industrial applications. Therefore, a $2 \%$ preload indicates a preload loss situation where preload loss should exhibit more mechanical backlash, increased pitted ball trace, lower stiffness, and lead the oscillatory position error problems. Additionally, the pretension and oil cooling system on the hollow ball screw were used for to achieve thermo compensation to improve positioning accuracy.

A framework for studying biological systems by a statistical physics approach is introduced via MSE to obtain useful measures for quantifying dynamical complexity. Thus, the development features of preload loss are mimicked here to determine the health of the ball screw via the MSE method based on complexity of mechanical concepts. Calculations of the EMD/HHT and MSE were performed using Visual Signal AnCAD Inc.

Temperature Distribution Study

In this study, the ball screws were driven at 300 $\mathrm{rpm}, 600 \mathrm{rpm}$ and $900 \mathrm{rpm}$ under $2 \%, 4 \%$ and $6 \%$ 
preload conditions with and without the oil cooling system and with or without the application of ball screw pretension. Therefore, we first investigate the temperature distribution of the whole hollow ball screw drive system.

Figure 5 presents thermal images which illustrate the temperature distribution of the hollow ball screw and the trend toward temperature balance due to the oil cooling effect. Experimental results show the ball nuts are dominated high temperatures with the ball screw due to contact with the balls. Temperatures rise steeply within one hour of high-speed motion without the oil cooling system. Figure 6 shows additional detail on the temperature distribution for contact between the ball nut and ball screw. At an ambient temperature of $24^{\sim} 25^{\circ} \mathrm{C}$, the temperature reaches a maximum of $41.6^{\circ} \mathrm{C}$ without the oil cooling system. Figure 7 shows temperature variations of the ball nut and ball screw with and without the oil cooling system for the $2 \%$ preload ball screw. As seen in Figure 8 , when the ball screw preload is increased to $6 \%$, the temperature variations of the ball nut and ball screw increased slightly due to the frictional force between the ball nut and the ball screw. Most of the temperature rise effect is caused contact between the rotating ball nut and the entire ball screw. Figure 9 shows temperature variations of the ball nut and the $6 \%$ preload ball screw with and without the oil cooling circulation with $20 \mu \mathrm{m}$ pretension. As compared with Figure 8 (i.e., without pretension), the use of lack of oil cooling during one hour of operation has little impact on the temperature distribution of the ball nut and ball screw. In summary, the temperature difference between Figures 8 and 9 is difficult to discern when the ball screw oil pretension is applied without cooling. Therefore, without cooling, the prognostic diagnosis for the positioning accuracy of the preload status is indistinguishable from that with cooling. The following section describes experiments to examine the ball screw pretension effects on position accuracy when the preloading of each ball screw is changed.
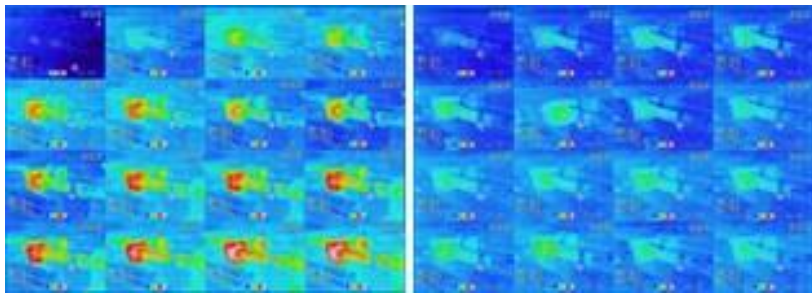

Figure 5. Temperature distribution of the ball screw without (left) and with (right) oil cooling for one hour of operation.

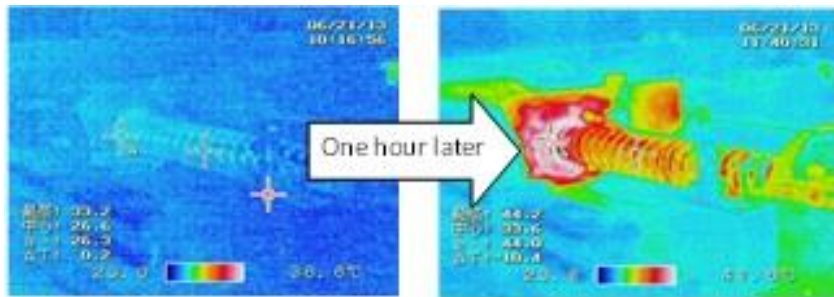

Figure 6. Temperature distribution of the ball screw and ball nut for one hour operation without oil cooling.

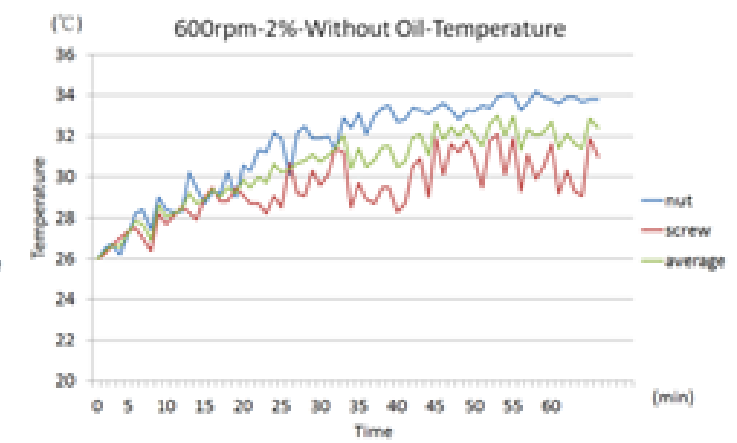

Figure 7. Temperature variations of the ball nut and ball screw with (left) and without (right) oil cooling system circulation for a $2 \%$ preload ball screw.
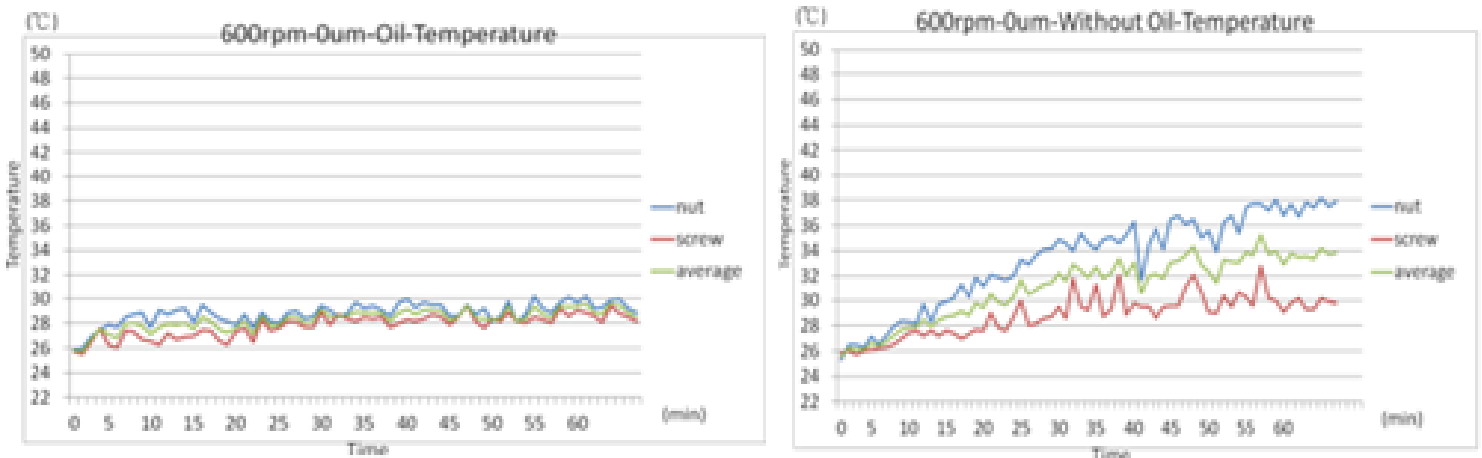

Figure 8. Temperature variations of the ball nut and ball screw with (left) and without (right) oil cooling circulation for $6 \%$ preload ball screw without pretension. 

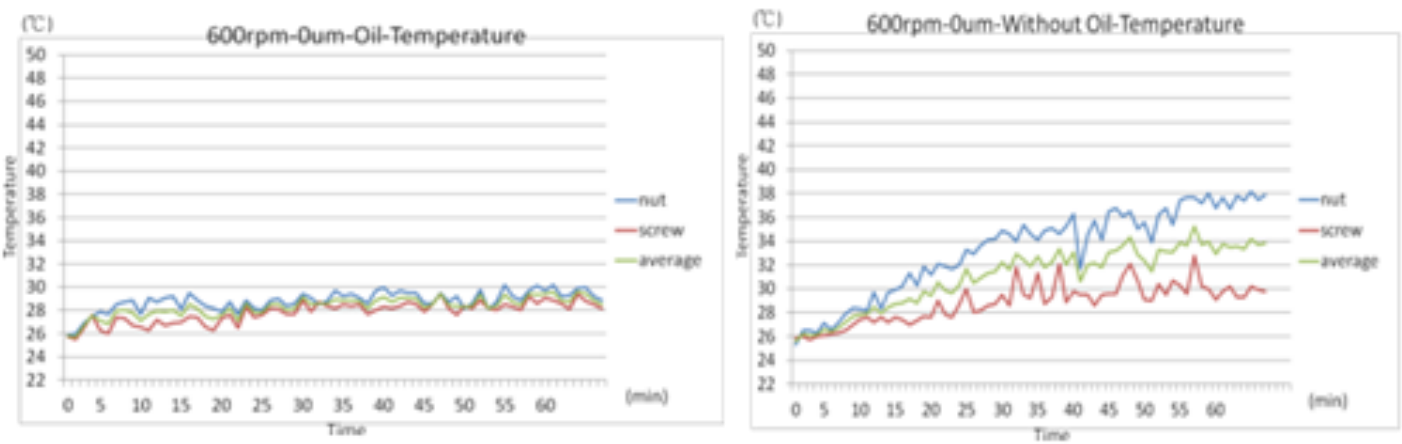

Figure 9. Temperature variations of the ball nut and ball screw with (left) and without (right) oil cooling circulation for $6 \%$ preload ball screw with 20 $\mu \mathrm{m}$ pretension.

Table 1. Improved positioning accuracy by point-to-point control based on different operating speed, preload and pretension conditions.

\begin{tabular}{|c|c|c|c|c|}
\hline $\begin{array}{l}\text { Operation Speed (rpm)/ } \\
\text { Improved positioning } \\
\text { accuracy (um) }\end{array}$ & $\begin{array}{c}2 \% \text { preload ball } \\
\text { screw without } \\
\text { pretension }\end{array}$ & $\begin{array}{c}4 \% \text { preload ball } \\
\text { screw without } \\
\text { pretension }\end{array}$ & $\begin{array}{l}4 \% \text { preload ball } \\
\text { screw with } 10 u m \\
\text { pretension }\end{array}$ & $\begin{array}{c}4 \% \text { preload ball } \\
\text { screw with 20um } \\
\text { pretension }\end{array}$ \\
\hline 300rpm & 1.790 & 1.203 & 1.145 & 1.246 \\
\hline 600rpm & 2.187 & 3.004 & 2.567 & 2.307 \\
\hline 900rpm & 3.712 & 2.663 & 2.714 & 3.221 \\
\hline $300 \mathrm{rpm} \rightarrow 600 \mathrm{rpm} \rightarrow 900 \mathrm{rpm}$ & 2.913 & 3.138 & 1.995 & 2.895 \\
\hline $\begin{array}{c}300 \mathrm{rpm} \rightarrow 600 \mathrm{rpm} \rightarrow 900 \mathrm{rpm} \\
\quad \rightarrow 600 \mathrm{rpm} \rightarrow 300 \mathrm{rpm}\end{array}$ & 1.187 & 1.138 & 1.134 & 1.444 \\
\hline
\end{tabular}

\section{Point-to-Point Positioning Accuracy}

Theoretically, the oil cooling system should reduce the positioning error to approximate the effect made by ball screw pretension when the machine tool is operated for long durations. Experiments were conducted to determine the effect of pretension or oil cooling using the linear scale feedback sensor (see Figure 10). The ball screw pretension was experimentally conducted by pulling the ball screw for different lengths. During rotation, the ball screw's concentric error was limited to less than $1 \mu \mathrm{m}$. Table 1 compares positioning accuracy with and without the oil cooling system. For example, the $1.79 \mu \mathrm{m}$ figure at the top left of the second column of Table 1 is calculated using the final position error with oil cooling circulation less the final position error without cooling. The machine table traveled back and forth a distance of $270 \mathrm{~mm}$ by repetitive motion. As shown in Table 1, the improved positioning accuracy for the $2 \%$ preload ball screw without pretension is approximately as same as for the $4 \%$ one. When $20 \mu \mathrm{m}$ pretension was applied to $4 \%$ preload ball screws, the positioning errors generally better than those made with $10 \mu \mathrm{m}$ pretension. Experimental results shown in Table 1 are within the set range of $2 \mu \mathrm{m}$ when different preload or pretension conditions were compared. This is close to the hardware reproducibility level. Oil cooling circulation and/or ball screw pretension are found to have no noticeable effect on the preload characteristics for point-to-point positioning. In addition, pretension had little impact on positioning accuracy. Future studies will assess the impact of oil cooling circulation and/or ball screw pretension on tracking error.

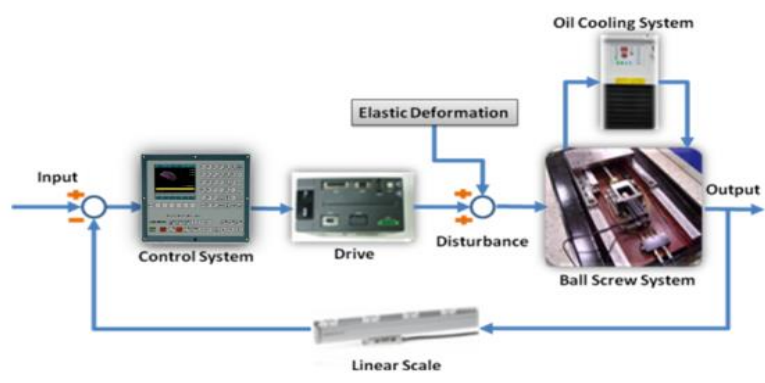

Figure 10. Feedback control block diagram of the single ball screw servo drive system with oil cooling and ball screw pretension.

\section{MSE Diagnosis}

In [10], a diagnostic method was presented to determine the characteristics of different preload levels based on servo motor current. Figure 11 illustrates the enlargement of the motor current profile of the $4 \%$ hollow ball screw with preload of $300 \mathrm{rpm}$ without pretension. Figure 12 provides a detailed look at the time-frequency spectrum via HHT, showing significant patterns of the current drive frequencies within the time domain. Note the many color strips in the time and frequency spectrum in the acceleration, constant speed and deceleration time zones. Such phenomena 

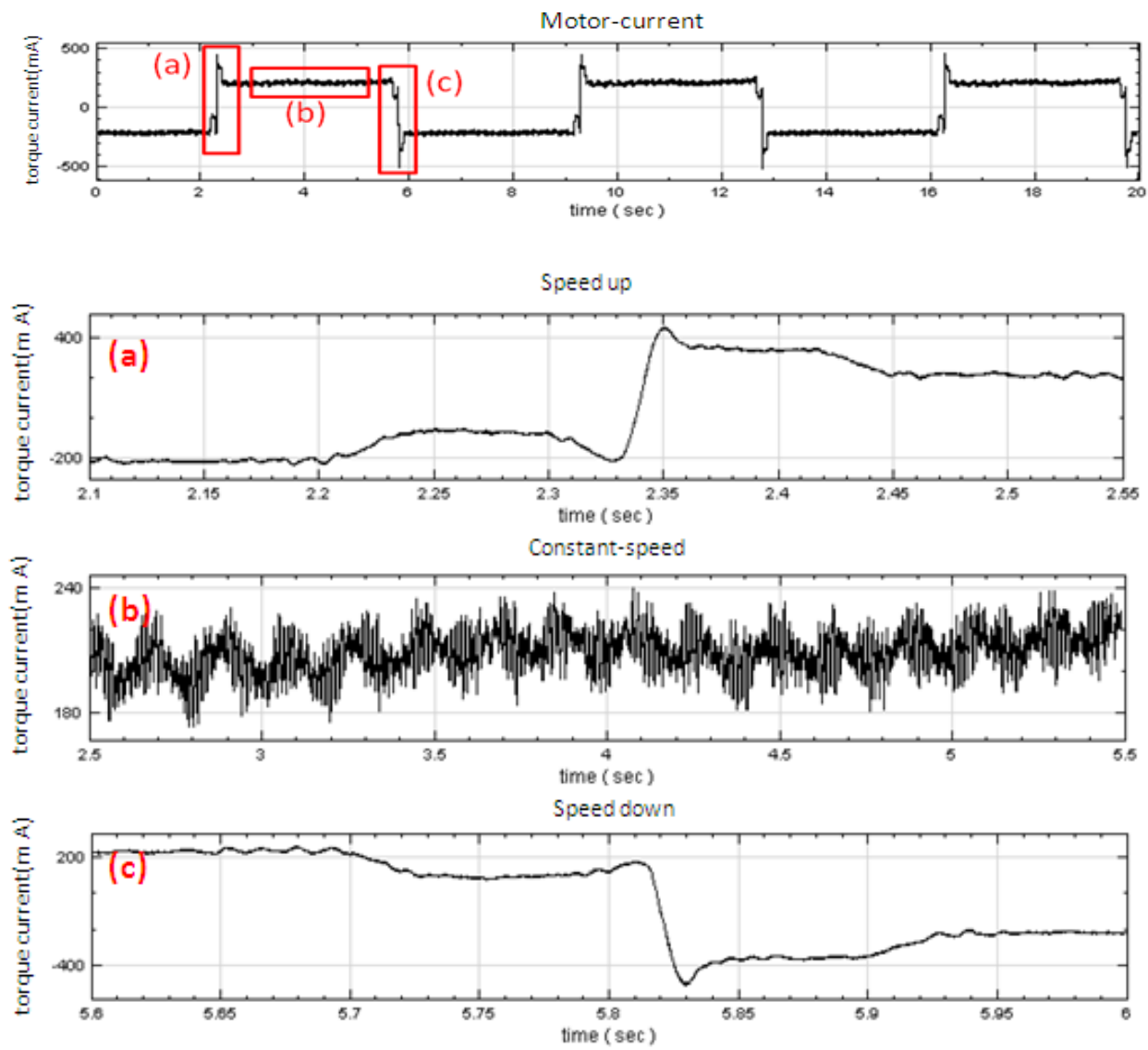

Figure 11. Profile of the motor current of a $4 \%$ ball screw motion based on a stroke of $270 \mathrm{~mm}$ by 300 rpm without pretension.

demonstrate the impact of oil cooling circulation or thermo suppression on the driving current frequencies by smoothing the friction of the ball contact with the nut, the ball rotating around the ball raceway, the ball circulating about the ball screw and the balls themselves spinning in contact with each other. Figures 13 and 14 respectively show the MSE plots of $600 \mathrm{rpm}$ based on $2 \%$ and $4 \%$ preload, respectively. As expected, it's conceivable that the MSE value without oil cooling is higher than that with oil cooling at three different zones given a constant rpm. The thermo expansion on frictional force is related to the actuated current fluctuation. Figure 15 shows the MSE plots of the $2 \%$ and $4 \%$ preload ball screws when speed is increased from $300 \mathrm{rpm}$ to $600 \mathrm{rpm}$ and then to 900rpm both with and without oil cooling. Oil cooling is seen to reduce the MSE value at different zones. Figure 16 shows the MSE plot for the $2 \%, 4 \%$ and $6 \%$ preloaded ball nut screws by changing the speed from 300rpm to 600rpm and then to $900 \mathrm{rpm}$ both with and without oil cooling. Experimental results show that, both with and without oil cooling, the MSE value of the $6 \%$ preload ball screw was higher than those of both the $4 \%$ and $2 \%$ ones when the machine stage was driven at variable speeds, especially as the machine speed accelerated. Experimental results show

www.ausmt.org

Copyright (C) 2014 International Journal of Automation and Smart Technology that a higher MSE value indicates a high degree of entropy, after which temperature increases with motion speed and the balls expand due to increased friction. This, in turn, increases the preload effect, resulting in a high complexity index. This corresponds to the mechanical complexity hypothesis associated with rich structural responses. Intuitively, as the preload loss occurs, the balls inside the ball nut and raceway of the ball screw create more rolling contact, along with increased random vibration motion and irregularity. Note that, even though the oil cooling was applied (bottom of Figure 16), the varying and increasing speeds caused a significant rise in temperature. The expansion of the balls, especially for larger-sized injected balls with the $6 \%$ preload ball screw, circulate through the entire ball nut loop within the ball screw raceway and then dominate the frictional force despite the use of the cooling system. The $2 \%$ preload balls are predicted to expand to the $4 \%$ standard size, thus producing thermo expansion effects and causing abrupt and irregular motor current during the MSE calculation. Experimental results revealed the temperature effect was more significant than that of the preloaded one when the machine tool was operated at varying speeds. 


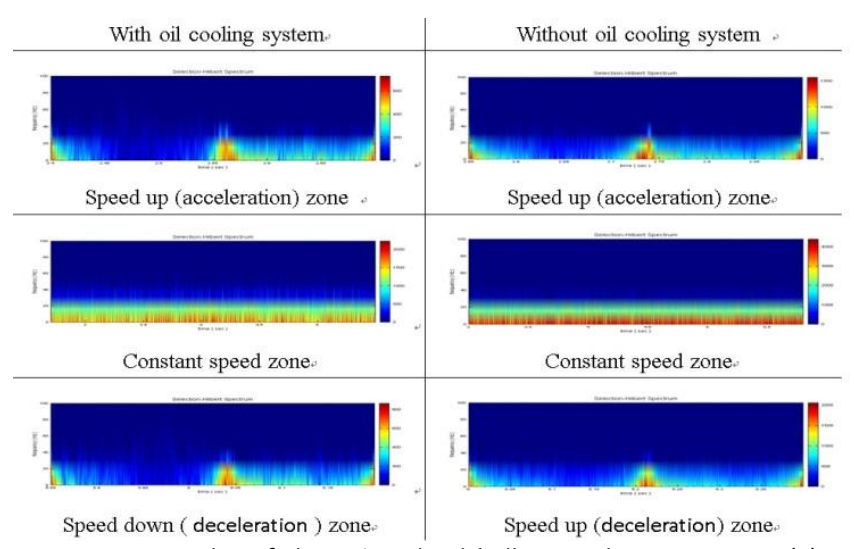

Figure 12. HHT plot of the $6 \%$ preload ball screw by $300 \mathrm{rpm}$ at (a) acceleration zone, (b) constant speed zone and (c) deceleration zone with and without oil cooling.

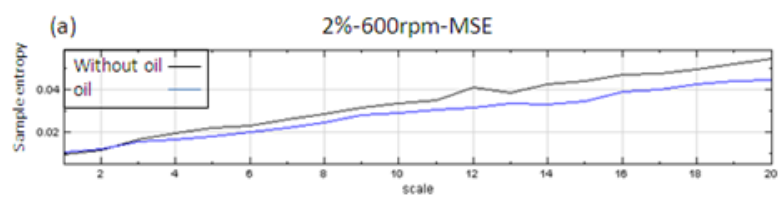

(b) 2\%-600rpm-MSE

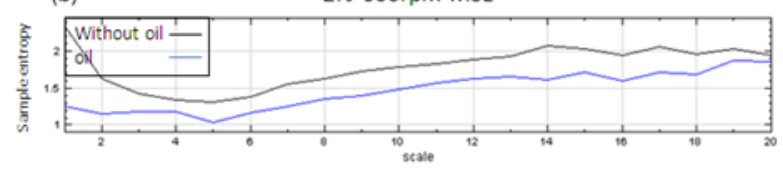

(c)

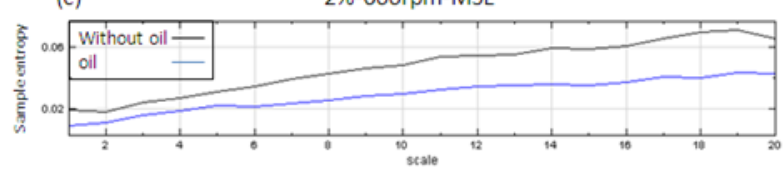

Figure 13. MSE plot of $2 \%$ preload ball screw by $600 \mathrm{rpm}$ at (a) acceleration zone, (b) constant speed zone and (c) deceleration zone with and without oil cooling.
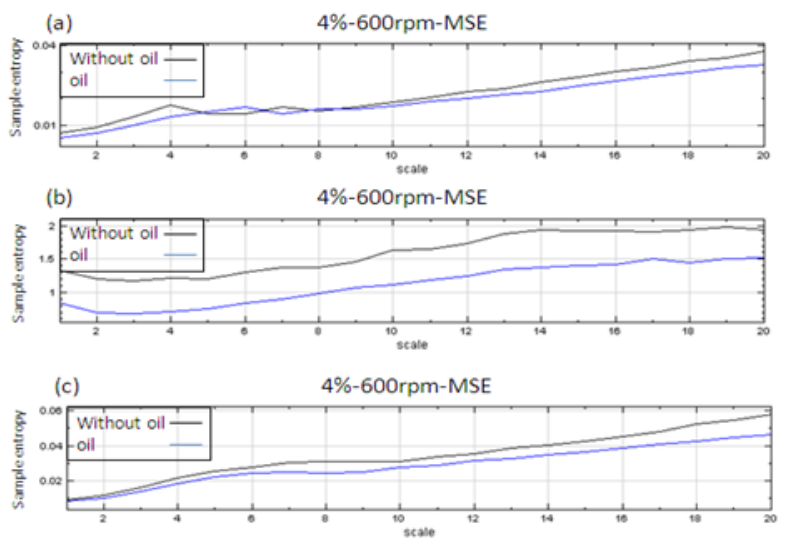

Figure 14. MSE plot of $4 \%$ preload ball screw by $600 \mathrm{rpm}$ at (a) acceleration zone, (b) constant speed zone and (c) deceleration zone with and without oil cooling.

In sum, the proposed method allows manufacturers to effectively identify potential preload loss and address the issue before it creates production problems. Experimental results suggest the approach offers promise for prognostic monitoring of ball nut preload status in industrial applications. The technique offers an inexpensive, robust and reliable means of diagnosing the condition of ball screws through the servo current signals, and can be easily integrated into future manufacturing processes.

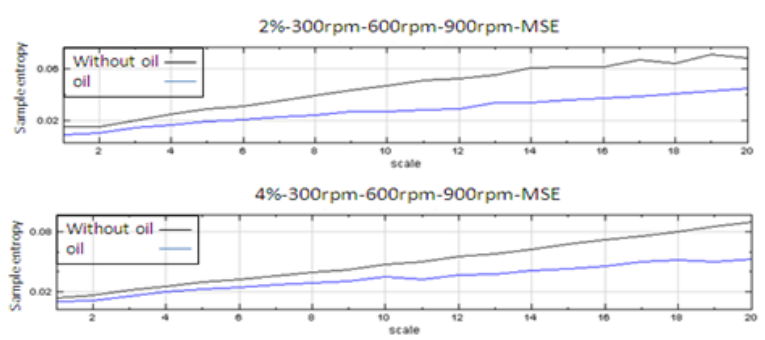

Figure 15 . MSE plots of $2 \%$ and $4 \%$ preload ball screws by changing the speed from 300 to 600 and then to $900 \mathrm{rpm}$ with and without oil cooling.

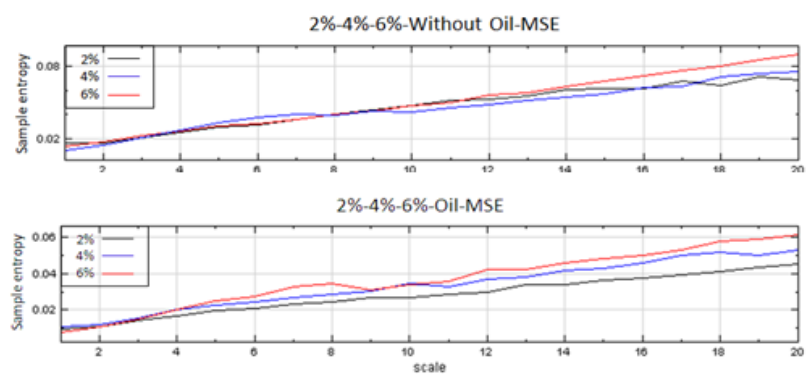

Figure 16. MSE plots of $2 \%, 4 \%$ and $6 \%$ preload ball screws by increasing the speed from 300rpm to 600rpm and then to $900 \mathrm{rpm}$ with and without oil cooling.

\section{Conclusion}

Signal analysis techniques are used to detect the preload status of hollow ball screw nuts in a machine tool feed drive table with an oil cooling system and ball screw pretension. Both the highly nonlinear processing $\mathrm{EMD} / \mathrm{HHT}$ and MSE methods are used to diagnose the machinery health status. A computational measure of MSE was used to dynamically quantify the hollow ball screw's complexity and identify the irregular development of the ball nut preload. The drive servo current signals of the preload features were extracted using significant patterns of the Hilbert-Huang spectrum. Compensated positioning technique by ball screw pretension and oil cooling was significantly contaminated for prognostic diagnosis. The signals regime of the speed zones were then discriminated by MSE, thus obtaining useful MSE measures reflecting the status of preload loss of the ball screw. Experimental results show a clear inclination towards complexity once the hollow ball screw nut develops a preload loss under constant speed command tests. Results also show that proposed method can ascertain the prognostic machinery state when the ball screw is in operation. 


\section{Acknowledgment}

This research was supported in part by the National Science Council, Taiwan under contract No. NSC-102-2221-E-018-008.

\section{References}

[1] C. Huang, S. Chen, H. Liou, W. Wang, L. Chen, T. Huang, and K. Hou, "A machine tool performance monitoring system for use on common hand-held devices," Journal of the Chinese Society of Mechanical Engineers, vol. 30, no. 4, pp. 323-331, 2009.

[2] H. Shimoda, "Stiffness analysis of ball screws--influence of load distribution and manufacturing error," International Journal of the Japan Society for Precision Engineering, vol. 33, no. 3, pp. 168-172, 1999.

[3] N. E. Huang, Z. Shen, S. R. Long, M. C. Wu, H. H. Shih, Q. Zheng, N.-C. Yen, C. C. Tung, and H. H. Liu, "The empirical mode decomposition and the hilbert spectrum for nonlinear and nonstationary time series analysis," Proceedings of the Royal Society A, vol. 454, pp. 903-995, 1998. doi: $10.1098 /$ rspa.1998.0193

[4] D. Gabor, "Theory of communication," Joint Information Exchange Environment, vol. 93, no. 26, pp. 429-457, 1946.
[5] J. Antonino-Daviu, P. J. Rodriguez, M. Riera-Guasp A. Arkkio, J. Roger-Folch, and R. B. Pérez, "Transient detection of eccentricity-related components in induction motors through the hilbert-huang transform," Energy Conversion and Management, vol. 50, no. 7, pp. 1810-1820, 2009 doi: 10.1016/j.enconman.2009.03.008

[6] Z. K. Peng, P. W. Tse, and F. L. Chu, "A comparison study of improved hilbert-huang transform and wavelet transform: Application to fault diagnosis for rolling bearing," Mechanical Systems and Signal Processing, vol. 19, no. 5, pp. 974-988, 2005. doi: $10.1016 /$ j.ymssp.2004.01.006

[7] M. Costa, A. L. Goldberger, and C. K. Peng, "Multiscale entropy analysis of biological signals," Physical review. E, Statistical, nonlinear, and soft matter physics, vol. 71, no. 2, pp. 021906, 2005. doi: 10.1103/PhysRevE.71.021906

[8] J. S. Richman and J. R. Moorman, "Physiological time-series analysis using approximate entropy and sample entropy," American journal of physiology. Heart and circulatory physiology, vol. 278, no. 6, pp. H2039-2049, 2000.

[9] S. M. Pincus, "Approximate entropy as a measure of system complexity," Proceedings of the National Academy of Sciences, vol. 88, pp. 2297-2301, 1991.

[10] Y. Huang and J. Chang, "Prognostic diagnosis of ball screw preload loss for machine tool through the hilbert-huang transform and multiscale entropy measure," Journal of the Chinese Society of Mechanical Engineers, vol. 32, no. 4, pp. 349-358, 2011. 